\title{
Organ Elasticity
}

National Cancer Institute

\section{Source}

National Cancer Institute. Organ Elasticity. NCI Thesaurus. Code C119332.

A quantitative measurement of an organ's ability to deform in response to physiological force and return to its normal shape by passive elastic recoil. 